\title{
A Proposal for Automatic Diabetes Food Information Display with Mobile Phone
}

\author{
*Mei-kui Zhang \\ Telemedicine Center \\ General Hospital of PLA \\ Beijing, China \\ zmk301@126.com
}

Jing-sheng $\mathrm{Hu}$

Department of Endocrinology

First Affiliated Hospital, General Hospital of PLA

Beijing, China

hjs304@yahoo.com.cn

\author{
Kun Peng \\ Department of Endocrinology \\ First Affiliated Hospital, General Hospital of PLA \\ Beijing, China
}

\author{
Ya-bin Li \\ Telemedicine Center \\ General Hospital of PLA \\ Beijing, China
}

\author{
Chen Jiang \\ Telemedicine Center \\ General Hospital of PLA \\ Beijing, China
}

\begin{abstract}
Dietary treatment is the basic therapy for diabetes, but how to do the right food intake is the biggest discouraging problem. We present a proposal for mobile phone diabetes food information display which can help determine the food composition and calories automatically from the clinical point of view with the mature communication technology. We analyzed the composite of the device, especially the key technical method, which is digital image recognition, three-dimensional image analysis, standard tables of food composition database and composite meals energy calculation auxiliary knowledge base. The device has technical feasibility and broad application prospects. It's combination of dietary treatment with smart mobile technology, which can provide great support to solve the problem of food energy assessment and analysis, not only for the single-ingredient food but also the composite meals. The proposal also provides further thinking about how to improve adherence to medical nutrition treatment in diabetes.
\end{abstract}

Keywords-dietary assessment; smart mobile phone; image analysis; medical nutrition therapy; device

\section{INTRODUCTION}

It is very important for guiding treatment of diabetic patients with non-invasive, continuous, automatic, dynamic disease monitoring. The accuracy and completeness of data has a significant role in understanding of disease, guiding doctors to adjust treatment, guiding patient's lifestyle improvements such as diet and exercise. For the techniques and instruments such as portable blood glucose meter, blood pressure meter, exercise housekeeper (multi-function pedometer), ECG monitoring have used more widely, but how to do the right food intake in front of the wide variety of food species is still a problem, especially for young people and middle-aged man who can't live regularly and healthy[1].

Conventional treatment is to enhance food education, and under the guidance of a registered dietitian to develop and monitor diet plan [2], but there are still many problems in implementation which caused a great increase in the prevalence of diabetes all over the world. Dietary treatment also known as "medical nutrition therapy" is the base treatment of diabetes, hyperlipidemia, obesity, hypertension and many other chronic diseases [3,4]. This paper discussed how to solve this problem by application of the automatic food information device with smart mobile technology.

\section{Mobile HeAlth And Diabetes}

Mobile health is a new and advancing field in telehealth. It can be used to transfer patient biometrics, to provide reminders to patients about medications or scheduled medical appointments, and to empower patients by sending out information such as the nearest location to get help or an update on diabetes management [5].

Preuveneers[6] has investigated how the mobile phone platform can contribute with individuals diagnosed of diabetes to handle their glucose in blood levels without resorting to no additional systems or without adding any additional activity sensors, such as pedometers or heartbeat monitors. Supervising the location and activity of the patient with the mobile phone, recognizing past behaviours and knowing the glucose in blood levels with context information and maintain stable of the blood glucose level.

\section{OUR PROPOSAl For CONSTRUCTION Of AutOMATIC FOOD INFORMATION DISPLAY}

We present our proposal for automatic food information display with mobile phones as follows:

\section{A. Application of digital image recognition technology [7] to identify what food is:}

Scanning of all the common used food to take images with which the application of computer-aided digital image recognition technology to determine the types of food. According to the shape and colors of food images assistant 
with manual choice, determine the types of food given for users to choose.

\section{B. Application of three-dimensional image analysis techniques [8] to determine the weight of food:}

Based on identifying the types of food, measure the volume of food, automatically analysis and calculates the food weight applying three-dimensional imaging technology.

\section{Standardized food composition table [9]:}

With the existing standardized national "Food Composition Table", which can be checked the common nutritional data systematically, link the database information with the image identification and measurement results automatically to get food calorie information displayed on the display screen.

\section{Single-ingredient food identification, assessment and energy analysis:}

According to this principle, the energy identification and analysis of single ingredient food is relatively easy and as follows: according to the images of acquired food, the device can discriminate the types of food and estimated the weight, and on the base of the food composition database, the device can calculate the single food detailed calories automatically.

\section{E. Compound food identification and energy analysis:}

Most food we take every day is very diversifying which we called composite meals. Composite meal is composed of a variety of single food by complex manufacture. Its caloric composition is widely affected not only by the complex and diverse composition of food but also by external personal habits (the proportion of ingredients, cooking conditions, etc.). So how to assess the energy of composite meals is still a major problem in medical nutrition treatment.

We design a composite meals energy calculation auxiliary knowledge base to solve the problem. The auxiliary knowledge base is used to store the proportion of single food in the composite meals which people often cooked. For example, the general quantity of flour, cheese, sausage and sauce which are used in the 100 grams of pizza will be stored in the auxiliary knowledge base in advance. If an individual eats a piece of pizza, the image of pizza should be captured and identified by using three-dimensional imaging digital camera firstly, and the weight of pizza can be calculated automatically. Then, the proportion of main raw material of pizza will be extracted from the auxiliary knowledge base for computing the respective weight of flour, cheese, sausage and sauce which are used in this pizza. Finally, the caloric of whole pizza can be calculated by combining the fat, protein, calories of single ingredient of pizza such as flour, which can be found in food hot scale.

In addition, the auxiliary knowledge base can also be customized and revised by an individual, the raw material proportion of pizza which we cooked can also be stored in auxiliary knowledge base, and revised according to the individual's eating habits constantly. So individuals can control personal diet more accurately. The process flow of composite meals energy analysis is shown in the Figure 1.

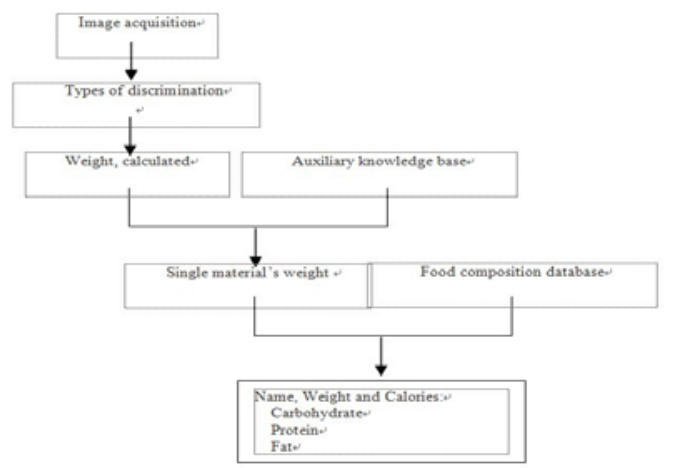

Figure 1. Framework proposal for composite meals energy analysis

\section{KEY TECHNICAL METHOD}

\section{A. Three-Dimensional Analysis for Digital Images}

The technology of creating a three-dimensional image by analyzing two-dimensional images is more and more common and convenient. Even by only one digital photograph, we can get the three-dimensional model[10]. We can take digital photos of food by cameras on the device at first, then we deal with the digital photos by a serial of algorithms including image grayed, image denoised, character information fetching and 3D recreation. At last, we can calculate the different component's volume.

\section{B. Design of Database}

The database includes the information as follows at least: auxiliary knowledge base data, food composition data, custom data and user's favorite data. The pattern of information storage could be designed as relative database based on EntityRelationship model, such as Access, MySQL, SQLServer, Oracle etc. And it could be designed as noSQL database, such as MongoDB, Voldemort, ESENT etc. The former are commonly used in various applications and softwares. And noSQL databases have the features, such as highly extensible and dealing with vast information. Some knowledge engineering tools choose noSQL database as the basis data store[11].

\section{TOWARDS A FRAMEWORK OF AUTOMATIC FOOD INFORMATION DISPLAY}

Mobile phone can be constructed as a platform for diabetes patient information analysis with function of information collection, storage, processing, analysis and display. Our proposal for automatic food information display with mobile phones consists of three parts, namely image capture device, the information processor and display which all can implement in a mobile phone. Image capture device that is a built-in three-dimensional imaging digital camera, which has characters of three-dimensional imaging, fixed focus, large aperture, and can form a fixed three-dimensional digital pixel images. Information processor can determine the possible types of food according to the captured digital photo. 
The food image is showed on the touch screen. The information processor can calculate the three-dimensional image volume and estimates the food weight, automatically calculated and display the caloric of food component on the screen according to the food department coefficient in the "Food Composition Table" and composite meals energy calculation auxiliary knowledge base.

\section{DISCUSSION}

\section{A. Diabetic diet in the treatment of diabetes}

Diabetes diet therapy, also known as "medical nutrition therapy", usually aided by a registered dietitian according to one on one guidance model with diabetic patients, which included nutrition education, nutrition assessment, nutrition diagnosis, nutrition intervention, nutrition monitoring and evaluation [3,12]. In the case of can't be fundamentally restore islet function in diabetes, whether type 1 or type 2 diabetes, diet therapy is the most important one of diabetes, and also the fundamental therapy of other treatments, including insulin therapy $[3,4]$. A large number of clinical trials have been clearly demonstrated that effective diet therapy can significantly reduce blood sugar levels, especially for patients with pre-diabetes and even reached good control of blood sugar with diet therapy alone[3,4,13]. Moreover, even if diabetes can be radically treatment and recovery in the future, a reasonable diet is the basis of prevention and treatment for many diseases, such as obesity, hypertension, hyperlipidemia, cardiovascular diseases, and even for tumors [14].

\section{B. Problems of diet therapy}

Dietary treatment is simple and effective, but it is still the biggest problem in diabetes treatment [15]. First, the diversify of diet type make it is difficult for everyone to grasp more fully and clearly, and the "one to one" approach is very difficult to turn to a registered dietitian for guidance each meal. Secondly, the complicated calculations of food calories lead to poor clinical implementation, such as in order to define the food component, need to search the "Food Composition Table" to find the food carbohydrate factor, and then need computing into a specific food weight according to how much carbohydrates to eat. Thirdly, different diet and living habits and compliance differences are all due to the difficulties of diet therapy.

\section{Feasibility Analysis}

Among the three technical elements that make up the system, three-dimensional digital image recognition technology and image analysis technology has been applied in other areas, the technology is relatively mature. The standard "Food composition table" is a database which contains a large amount of food information for application. Meanwhile, the auxiliary knowledge base is based on mature database technology, so the implementation of the system is feasible.

\section{Technical difficulties and problems}

The digital image recognition and analysis technology has been used widely, but vast applications in a variety of food is rare, especially for the determination of size and weight maybe has large problems and errors. Secondly, the analysis of composite meals calories needs to realized gradually with the standards implementation of the complex food.

In summary, the system has technical feasibility and broad application prospects in the treatment of many chronic diseases, such as diabetes, hyperlipidemia, obesity and hypertension. The device can identify the food species and display it's energy components automatically. It's combination in dietary treatment and computer information technology, which can solve the problem of food energy assessment and analysis, not only for the single-ingredient food but also the composite meals. The system provided further thinking of technical support in the diet therapy of diabetes.

\section{REFERENCES}

[1] Sanjeev N. Mehta, Nicolle Quinn, Lisa K. Volkening, Lori M.B. Laffel, "Impact of Carbohydrate Counting on Glycemic Control in Children With Type 1 Diabetes,” Diabetes Care. Vol. 32(6), pp:1014-16, 2009.

[2] Sara F. Morris, and Judith Wylie-Rosett, "Medical Nutrition Therapy: A Key to Diabetes Management and Prevention," Clinical Diabetes. Vol. 28(1), pp: 12-18, 2010.

[3] Joyce Green Pastors, Hopewarshaw, Anne Daly, Marion Franz, Karmeen Kulkarni, "The Evidence for the Effectiveness of Medical Nutrition Therapy in Diabetes Management," Diabetes Care.Vol. 25(3), pp:608-613, 2002.

[4] Franziska K. Bishop, David M. Maahs, Gail Spiegel, Darcy Owen, Georgeanna J. Klingensmith, Andrey Bortsov, Joan Thomas and Elizabeth J. Mayer-Davis, "The Carbohydrate Counting in Adolescents With Type 1 Diabetes (CCAT) Study,” Diabetes Spectrum. Vol. 22(1), pp:56-62, 2009.

[5] Jason Goldwater and Yael Harris, "Using Technology to Enhance the Aging Experience: A Market Analysis of Existing Technologies,” Ageing Int. Vol. 36, pp:5-28, 2011.

[6] Preuveneers, D, Berbers, Y, "Mobile phones assisting with health self-care: a diabetes case study," In: Proceedings of $10^{\text {th }}$ internationak Conference on Human Computer interaction with Mobile Devices and Services, MobileHCI 2008, Amsterdam, The Netherlands, September 2-5, 2008, pp. 177-186. ACM, New York(2008).

[7] Fengqing Zhu, Marc Bosch, Carol J. Boushey, and Edward J. Delp, "An Image Analysis System for Dietary Assessment and Evaluation,” Proc Int Conf Image Proc. 2010: 1853-1856. doi:10.1109/ICIP.2010.5650848.

[8] Rick Weiss, MS, “Automatic Food Documentation and Volume Computation using Digital Imaging and Electronic Transmission,” J Am Diet Assoc. Vol. 110(1), pp: 42. 2010. doi:10.1016/j.jada.2009.10.011.

[9] Chinese Center for Disease Control and Prevention Institute for nutrition and food safety. China Food Composition Table. Peking University Medical Press.

[10] Guangmin Sun, Lei Xu, Deming Chen, Gang Li, Jing Wang, "A System Scheme of 3D Object Reconstruction From Single 2D Graphics Based on Neural Networks," Proceedings of 6th IEEE international Conference on Industrial Informatics(IEEE INDIN 2008), 2008. 7, Vol. 1-3, pp:10811085.

[11] Russ TA, Ramakrishnan C, Hovy EH, Bota M, Burns GA, "Knowledge engineering tools for reasoning with scientific observations and interpretations: a neural connectivity use case,” BMC Bioinformatics. Vol. 22(12), pp:351, Aug 2011.

[12] M J Franz, H Warshaw, A E Daly, J Green-Pastors, M S Arnold, J Bantle, "Evolution of diabetes medical nutrition therapy," Postgrad Med J. Vol. 79, pp:30-35, 2003. doi:10.1136/pmj. 79.927.30 
[13] Claudio Maffeis1 and Leonardo Pinelli, "Teaching children with diabetes about adequate dietary choices," British J Nutrition. Vol. 99(Suppl. 1), pp: S33-S39, 2008.

[14] Paul S. Mead, Laurence Slutsker, Vance Dietz, Linda F. McCaig, Joseph S. Bresee, Craig Shapiro,et al, "TauxeFood-
Related Illness and Death in the United States,” Emerging Infectious Diseases.Vol. 5(5), pp:607-625, 1999.

[15] Diane M. Reader, "Medical Nutrition Therapy and Lifestyle Interventions,” Diabetes Care. Vol. 30 (Suppl. 2), pp:S188-S193, 2007 